cases more than 3 weeks after the operation. The effect of thalamic pulvinotomy is most successful to the cases with severe chronic pain causing infiltrating or metastatic cancer (all of the cases required narcotics) and cerebrovascular accident ( 2 cases required narcotics). There was no recurrence in these 10 cases. From these facts, pulvinar thalamotomy have excellent effect for severe chronic pain require narcotics to relieve the pain.

We have had several autopsy cases, one of which was investigated anatomically and histologically and proved the invased area was located in the nucleus pulvinaries only.

\title{
19. Stereotaxic Thalamotomy for Intractable Pain. Study of Clinicopathological Correlation for the Best Target Point
}

\author{
Kenichi Uemura and E. S. WatKIns \\ 2nd Surgical Department, School of Medicine, Chiba University \\ Department of Neurosurgery, State University of New York, Upstate Medical Center, \\ Syracuse, New York, U.S.A.
}

Stereotaxic thalamotomy for intractable pain of various etiology has been performed on 29 cases with the follow-up length ranging from one month to six years. It was attempted to destroy the posterior, medial and inferior part of the centrum medianum with our average target point at $6 \mathrm{~mm}$ lateral from the midline and at $18 \mathrm{~mm}$ posterior from the posterior rim of the foramen of Monro along the line between the foramen of monro and the posterior commissure, and at $1 \mathrm{~mm}$ below this line. Electrical stimulation was used to control the position of the electrode tip during surgery.

In 9 cases who died of malignant diseases, the histological verification of the lesion was carried out to correlate with the clinical results of pain relief. In 7 cases with good pain relief, the lesion involved the almost entire parafascicular nucleus and the inferior half of the centrum medianum, completely interrupting the afferent fibers to the centrum medianum at its posterior portion, except in one case in which the lesion was only about $2 \mathrm{~mm}$ in diameter situated within the capsule ventral to the centrum medianum but interrupting all the afferent fibers to the centrum medianum where they are confluent before fanning out into the internal medullary lamina. In one case with partial pain relief, the lesion was a little too medial to leave the lateral half of the posterior inferior portion of the centrum medianum intact, and in the other case with no effect, the lesion involving the parafascicular nucleus and the inferior half of the centrum medianum was stoo anterior, leaving the posterior portion of the centrum medianum unaffected. These results strongly indicate that the lesion is best placed within the capsule sventral to the posterior portion of the centrum medianum so as to interrupt all 
the afferent fibers to the posterior portion of the centrum medianum and other intralaminar nuclei, if one wishes to obtain an excellent pain relief with a smallest lesion.

\title{
20. The Operative Indication of the CEM-Thalamotomy for the Relief of Intractable Pain
}

\author{
Miyoshi Urabe, Takashi TsuboKawa, Makoto Kikuchi, Satoru Kadoya, \\ Yoh Watanabe, Noboru Hamabe, Takeshi Ohtani, Haruhide Itoh, \\ Syuji Asano, Masaki Kidani, Takeshi Terauchi, Syogo Kuze, \\ Kunishige Watanabe and Hirokatsu Kitsukawa \\ Department of Surgery, School of Medicine, University of Kanazawa
}

We deviced a stereotaxic operation, CEM-Thalamotomy to make a circumscribed lesion involving the posteroventral part of the CEM (nucleus centrum medianum), for a relief of intractable pain.

The CEM-Thalamotomy were performed on 53 cases complaining of intractable pain which included 8 cases operated by Tsubokawa in Atlanta (VAH). According to analysis on the clinical course of all cases which were performed the CEM-Thalamotomy, the operative indication were as follows:

1) In the cases of visceral pain, bilateral CEM-Thalamotomy might produce sufficient effect on the pain, but in some case, somatic pain that is located on one side of body is relieved by the unilateral lesion.

2) The CEM-Thalamotomy can be used even on the cases with poor risk.

However, there is same limitation as the stereotaxic VL-tomy as there is more frequently brain metastasis, ventricular dilatation and venous stasis of the neck in terminal cancer patient.

3) According to our follow-up results, striking effect on pain caused by benign disease were observed more than three years and same effects on pain caused by malignant disease were observed for one to four months until death by original disease.

4) There is absolute contraindicative cases who have drug intoxication or psychiatric disorder, because the effect of the CEM-Thalamotomy and side effect are not correctly detected. 